\title{
Application of Homotopy Analysis Method to the Unsteady Squeezing Flow of a Second-Grade Fluid between Circular Plates
}

\author{
Mohammad Mehdi Rashidi, ${ }^{1}$ Abdul Majid Siddiqui, ${ }^{2}$ \\ and Mostafa Asadi ${ }^{1}$ \\ ${ }^{1}$ Department of Mechanical Engineering, Faculty of Engineering, Bu Ali Sina University, \\ P.O. Box 65175-4161, Hamedan, Iran \\ ${ }^{2}$ Department of Mathematics, Pennsylvania State University, York Campus, York, PA 17403, USA \\ Correspondence should be addressed to Mohammad Mehdi Rashidi,mm_rashidi@yahoo.com
}

Received 17 September 2010; Accepted 23 November 2010

Academic Editor: Victoria Vampa

Copyright (C) 2010 Mohammad Mehdi Rashidi et al. This is an open access article distributed under the Creative Commons Attribution License, which permits unrestricted use, distribution, and reproduction in any medium, provided the original work is properly cited.

\begin{abstract}
We investigated an axisymmetric unsteady two-dimensional flow of nonconducting, incompressible second grade fluid between two circular plates. The similarity transformation is applied to reduce governing partial differential equation (PDE) to a nonlinear ordinary differential equation (ODE) in dimensionless form. The resulting nonlinear boundary value problem is solved using homotopy analysis method and numerical method. The effects of appropriate dimensionless parameters on the velocity profiles are studied. The total resistance to the upper plate has been calculated.
\end{abstract}

\section{Introduction}

Squeezing flows are induced by externally normal stresses or vertical velocities by means of moving boundary. Squeezing flows have many applications in food industry, especially in chemical engineering. Some practical examples of squeezing flow include polymer processing, compression and injection molding. In addition, the lubrication system can also be modeled by squeezing flows. The study of squeezing flows has its origins in the 19th century and continues to receive considerable attention due to the practical applications in physical and biophysical areas. Stefan [1] published a classical paper on squeezing flow by using lubrication approximation. Such types of flow exist in lubrication when there is squeezing flow between two parallel plates. The tackiness of liquid adhesives also reflects squeeze film effects [2]. The squeeze film geometry has been studied extensively 
since 1947. Other applications in the biomechanics area relate to squeezing flow between parallel plates and the alternation between contraction and expansion of the blood vessels. In addition, polymer extrusion processes are modeled using squeezing flow of viscous fluids [3]. The squeezing flow between parallel plates when the confining walls have a transverse motion has great importance in hydrodynamic lubrication theory Langlois [4] and Salbu [5] have analyzed isothermal compressible squeeze films neglecting inertial effects. Thorpe [6] presented an explicit solution of the squeeze flow problem taking inertial terms into account. Later, P. S. Gupta and A. S. Gupta [7] showed that the solution given by [6] fails to satisfy the boundary conditions. Gupta solution, however, is restricted only to small Reynolds number. Squeeze film between two plane annuli with fluid inertia effects has been studied by Elkouh [8]. Some numerical solutions of squeezing flow between parallel plates has been conducted by Verma [9] and later by Singh et al. [10]. In addition, Hamza [11] has considered suction and injection effects on flow between parallel plates reflecting squeezing flow. Further work on second grade fluid flow between parallel plates has been carried out by Rajagopal and Gupta [12] and by Dandapat and Gupta [13]. Both papers consider the problem of flow between rotating parallel plates. Barron and Wiley [14] have also considered this fluid model to extend the Newtonian flow theory for slender bodies in a dusty gas. This fluid model has also been considered even in flow of a dusty liquid. For example, Debnath and Ghosh $[15,16]$ used this fluid model to study the flow of dusty fluids in rotating and oscillating cases, in the presence of magnetic fields. Hamdan and Barron [17] used this fluid model to analyse the steady squeezing flow of incompressible dusty fluids between two cylindrical plates. The theoretical and experimental studies of squeezing flows have been conducted by many researchers [18-28]. In this work the unsteady 2-dimensional flow of nonconducting, incompressible second grade fluid between circular plates that are moving symmetrically about the line of axial symmetry and giving rise to the squeezing flow is studied. The unsteady equations of motion are reduced to a single nonlinear fifth-order ordinary differential after employing a similarity transformation. The resulting nonlinear boundary value problem is solved with homotopy analysis method (HAM) and numerical method.

Most scientific problems and phenomena are modeled by nonlinear ordinary or partial differential equations. In most cases, these problems do not have precise analytic solution. Recently, much attention has been devoted to the newly developed methods to construct approximate analytic solutions of nonlinear equations. The HAM is developed in 1992 by Liao [28-35] and Rashidi et al. [36-38]. Liao applied this method to solve many types of nonlinear equations in science and engineering and then, this method has been successfully applied to solve many other nonlinear evolution equations. In recent years, the application of HAM in nonlinear problems has been developed by scientists and engineers. We know all perturbation methods require small parameter in nonlinear equation and the approximate solutions of equation containing this parameter are expressed as series expansions in the small parameter. Selection of small parameter requires a special skill. A proper choice of small parameter gives acceptable results, while an improper choice may result in incorrect solutions. The HAM, does not require a small parameter in equation modeling phenomena. Briefly speaking, Liao's method is a universal one which can solve various kinds of nonlinear equations, thus many researchers applied this method to various linear and nonlinear problems. A substantial amount of research work has been invested in the study of linear and nonlinear systems of PDEs. 


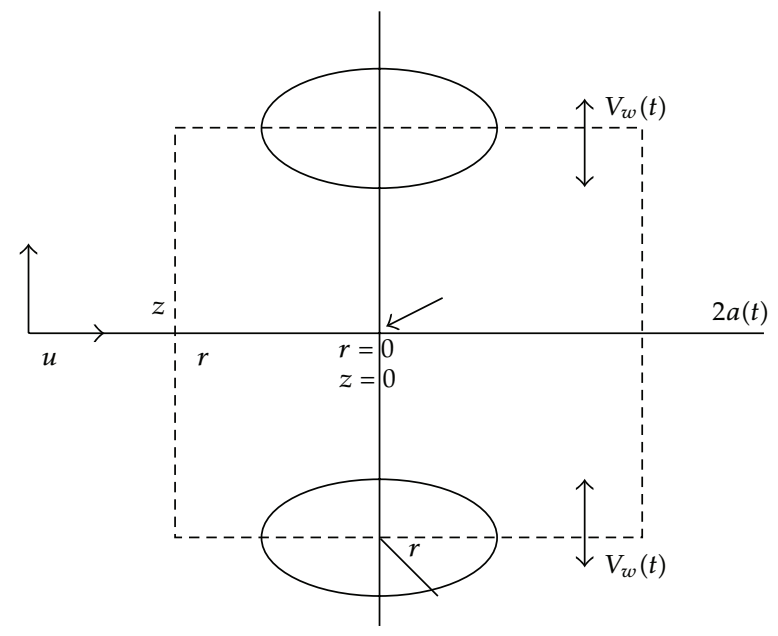

Figure 1: Geometry of the problem.

In Sections 2 and 3 description and mathematical formulation of the problem is presented. Sections 4 and 5 discussed the methods of solution of the problem. The results are discussed in Section 6 and Section 7 concludes finding of the present work.

\section{Problem Description}

The unsteady two-dimensional squeezing flow of nonconducting, incompressible second grade fluid between two circular plates is studied. The distance between the plates at any time $t$ is $2 a(t)$. The central axis of the channel is taken as the $r$-axis and $z$-axis is normal to it. It is assumed that the circular plates move symmetrically with respect to the central axis $z=0$ and flow is axisymmetric about $r=0$. The velocity components along the radial and axial directions are $u(r, z, t)$ and $w(r, z, t)$, respectively (Figure 1$)$. The fluid is assumed to have density $\rho$ and kinematics viscosity $v$.

\section{Mathematical Formulation}

The basic equations governing the motion of a homogeneous incompressible second grade fluid neglecting the thermal effects are [37]

$$
\begin{gathered}
\frac{1}{r} \frac{\partial}{\partial r}(r u)+\frac{\partial}{\partial z}(w)=0 \\
\rho\left(\frac{\partial u}{\partial t}+u \frac{\partial u}{\partial r}+w \frac{\partial u}{\partial z}\right)=-\frac{\partial P}{\partial r}+\mu\left(\frac{\partial^{2} u}{\partial r^{2}}+\frac{1}{r} \frac{\partial u}{\partial r}-\frac{u}{r^{2}}+\frac{\partial^{2} u}{\partial z^{2}}\right)+F_{r} \\
\rho\left(\frac{\partial w}{\partial t}+u \frac{\partial w}{\partial r}+w \frac{\partial w}{\partial z}\right)=-\frac{\partial P}{\partial z}+\mu\left(\frac{\partial^{2} w}{\partial r^{2}}+\frac{1}{r} \frac{\partial w}{\partial r}+\frac{\partial^{2} w}{\partial z^{2}}\right)+F_{z}
\end{gathered}
$$


where

$$
T=-p I+\mu A_{1}+\alpha_{1} A_{2}+\alpha_{2} A_{1}^{2}
$$

where $V$ is the velocity vector, $P$ is the pressure, $T$ is the stress tensor, $\mu$ is the coefficient of viscosity and $\alpha_{1}$ and $\alpha_{2}$ are material constants. The Rivlin-Ericksen tensors $A_{i}$ are defined as

$$
\begin{gathered}
A_{1}=\nabla V+(\nabla V)^{T}, \\
A_{2}=\frac{d A_{1}}{d t}+A_{1}(\nabla V)+(\nabla V)^{T} A_{1} .
\end{gathered}
$$

Now we formulate the equations of motion for unsteady two dimensional flow. Assuming that

$$
V=[u(r, z, t), 0, w(r, z, t)]
$$

and introducing the vorticity function $\Omega(r, z, t)$ and generalized pressure gradient $h(r, z, t)$ as

$$
\begin{gathered}
\Omega=\frac{\partial w}{\partial r}-\frac{\partial u}{\partial z} \\
h=\frac{\rho}{2}\left(u^{2}+w^{2}\right)+p-\alpha_{1}\left(w\left(\frac{\partial}{\partial r}+\frac{1}{r}\right)-u \frac{\partial}{\partial z}\right) \Omega-\frac{1}{4}\left(3 \alpha_{1}+2 \alpha_{2}\right)\left(A_{1}^{2}\right),
\end{gathered}
$$

where

$$
A_{1}^{2}=\left[4\left(\frac{\partial u}{\partial r}\right)^{2}+4\left(\frac{\partial w}{\partial z}\right)^{2}+4\left(\frac{u}{r}\right)^{2}+2\left(\frac{\partial u}{\partial z}+\frac{\partial w}{\partial r}\right)^{2}\right]
$$

We find that the equations of motion (3.1) and (3.2) reduce to

$$
\begin{aligned}
& \frac{\partial u}{\partial r}+\frac{u}{r}+\frac{\partial w}{\partial z}=0 \\
& \frac{\partial h}{\partial r}+\rho\left(\frac{\partial u}{\partial t}-w \Omega\right)=-\left(\mu+\alpha_{1} \frac{\partial}{\partial t}\right) \frac{\partial \Omega}{\partial t}-\alpha_{1} w\left(\left(\frac{\partial^{2}}{\partial r^{2}}+\frac{1}{r} \frac{\partial}{\partial r}+\frac{\partial^{2}}{\partial z^{2}}\right) \Omega-\frac{\Omega}{r^{2}}\right) \\
&+\left(\alpha_{1}+\alpha_{2}\right)\left(\frac{2}{r} \frac{\partial}{\partial z}(u \Omega)+\frac{\Omega^{2}}{r}\right) \\
& \frac{\partial h}{\partial z}+\rho\left(\frac{\partial w}{\partial t}+u \Omega\right)=\left(\mu+\alpha_{1} \frac{\partial}{\partial t}\right)\left(\frac{\partial \Omega}{\partial r}+\frac{\Omega}{r}\right)+\alpha_{1} u\left(\left(\frac{\partial^{2}}{\partial r^{2}}+\frac{1}{r} \frac{\partial}{\partial r}+\frac{\partial^{2}}{\partial z^{2}}\right) \Omega-\frac{\Omega}{r^{2}}\right) \\
&-\left(\alpha_{1}+\alpha_{2}\right)\left(\frac{2}{r} \frac{\partial}{\partial z}(u \Omega)\right)
\end{aligned}
$$


where $\nabla^{2}=\partial^{2} / \partial r^{2}+(1 / r)(\partial / \partial r)+\partial^{2} / \partial z^{2}$ is Laplacian operator.

Eliminating the generalized pressure $h$ between (3.10) and (3.11) to obtain

$$
\begin{aligned}
\rho\left(\frac{\partial \Omega}{\partial t}+\right. & \left.\left(u \frac{\partial}{\partial r}+w \frac{\partial}{\partial z}-\frac{u}{r}\right) \Omega\right) \\
= & \left(\mu+\alpha_{1} \frac{\partial}{\partial t}\right)\left(\left(\frac{\partial^{2}}{\partial r^{2}}+\frac{1}{r} \frac{\partial}{\partial r}+\frac{\partial^{2}}{\partial z^{2}}\right) \Omega-\frac{\Omega}{r^{2}}\right) \\
& +\alpha_{1}\left(u \frac{\partial}{\partial r}+w \frac{\partial}{\partial z}-\frac{u}{r}\right)\left(\left(\frac{\partial^{2}}{\partial r^{2}}+\frac{1}{r} \frac{\partial}{\partial r}+\frac{\partial^{2}}{\partial z^{2}}\right) \Omega-\frac{\Omega}{r^{2}}\right) \\
& -2 \frac{\left(\alpha_{1}+\alpha_{2}\right)}{r}\left(\left(\frac{\partial^{2}}{\partial r^{2}}-\frac{1}{r} \frac{\partial}{\partial r}+\frac{\partial^{2}}{\partial z^{2}}\right) u \Omega+\frac{1}{2} \frac{\partial}{\partial z}\left(\Omega^{2}\right)\right) .
\end{aligned}
$$

The boundary conditions on $u(r, z, t)$ and $w(r, z, t)$ are

$$
\begin{gathered}
u(r, z, t)=0, \quad w(r, z, t)=v_{w}(t), \quad z=a, \\
w(r, z, t)=0, \quad \frac{\partial u(r, z, t)}{\partial z}=0, \quad z=0,
\end{gathered}
$$

where $v_{w}(t)=d a / d t$ denotes the velocity of the plates. The first two conditions are due to the no-slip condition at the upper plate and the remaining two follow from the symmetry of the flow at $z=0$.

If the dimensionless variable $\eta=z / a(t)$ is introduced, (3.6), (3.9), and (3.12) become

$$
\begin{gathered}
\frac{\partial w}{\partial r}-\frac{\partial u}{a \partial \eta}=\Omega \\
\frac{\partial u}{\partial r}+\frac{u}{r}+\frac{\partial w}{a \partial \eta}=0, \\
\rho\left(\frac{\partial \Omega}{\partial t}+\left(u \frac{\partial}{\partial r}+w \frac{\partial}{a \partial \eta}-\frac{u}{r}\right) \Omega\right) \\
=\left(\mu+\alpha_{1} \frac{\partial}{\partial t}\right)\left(\left(\frac{\partial^{2}}{\partial r^{2}}+\frac{1}{r} \frac{\partial}{\partial r}+\frac{\partial^{2}}{\partial z^{2}}\right) \Omega-\frac{\Omega}{r^{2}}\right) \\
+\alpha_{1}\left(u \frac{\partial}{\partial r}+w \frac{\partial}{a \partial \eta}-\frac{u}{r}\right)\left(\left(\frac{\partial^{2}}{\partial r^{2}}+\frac{1}{r} \frac{\partial}{\partial r}+\frac{\partial^{2}}{\partial z^{2}}\right) \Omega-\frac{\Omega}{r^{2}}\right) \\
-2 \frac{\left(\alpha_{1}+\alpha_{2}\right)}{r}\left(\left(\frac{\partial^{2}}{\partial r^{2}}-\frac{1}{r} \frac{\partial}{\partial r}+\frac{\partial^{2}}{\partial \eta^{2}}\right) u \Omega+\frac{1}{2 a} \frac{\partial}{\partial \eta}\left(\Omega^{2}\right)\right) .
\end{gathered}
$$


The boundary conditions on $u(r, z, t)$ and $w(r, z, t)$ are

$$
\begin{gathered}
u(r, \eta, t)=0, \quad w(r, \eta, t)=v_{w}(t), \quad \eta=1 \\
w(r, \eta, t)=0, \quad \frac{\partial u(r, \eta, t)}{\partial \eta}=0, \quad \eta=0 .
\end{gathered}
$$

If we define the velocity components as follows Singh et al. [10] and Birkhoff [39]

$$
u=\frac{-r}{2 a(t)} v_{w}(t) f^{\prime}(\eta), \quad w=v_{w}(t) f(\eta)
$$

It follows that (3.14) takes the form

$$
\Omega=\frac{r}{2 a(t)^{2}} v_{w}(t) f^{\prime \prime}(\eta)
$$

substituting (3.18) and (3.19) in (3.15) and (3.16), we find that the continuity equation is identically satisfied and (3.16) becomes

$$
\begin{aligned}
& \frac{a v_{w}}{v}\left(f f^{\prime \prime \prime}-2 f^{\prime \prime}-\eta f^{\prime \prime \prime}\right)+\frac{a\left(d v_{w} / d t\right)}{v v_{w}} f^{\prime \prime} \\
& \quad=f^{i v}+\frac{\left(\alpha_{1}+\alpha_{2}\right) v_{w}}{\mu a}\left(2 f^{\prime \prime} f^{\prime \prime \prime}+f^{\prime} f^{i v}\right)+\frac{\alpha_{1} v_{w}}{\mu a}\left(f f^{v}-\eta f^{v}-4 f^{i v}+\frac{a\left(d v_{w} / d t\right)}{v v_{w}^{2}} f^{i v}\right),
\end{aligned}
$$

where prime denotes differentiation with respect to $\eta$. The boundary conditions are determined from (3.17), (3.18), and (3.19) to be

$$
\begin{array}{ll}
f(1)=1, & f^{\prime}(1)=0, \\
f(0)=0, & f^{\prime \prime}(0)=0 .
\end{array}
$$

Thus for a similarity solution we define

$$
\begin{gathered}
\frac{a v_{w}}{v}=R_{e}, \quad \frac{a^{2}\left(d v_{w} / d t\right)}{v v_{w}}=R_{e} Q, \\
\frac{\alpha_{1} v_{w}}{\mu a}=W_{e 1}, \quad \frac{\alpha_{2} v_{w}}{\mu a}=W_{e 2},
\end{gathered}
$$

where $R_{e}, Q$ and $W_{e 1}$ and $W_{e 2}$ are functions of $t$ but for a similarity solution $R_{e}, Q, W_{e 1}$, and $W_{e 2}$ are considered to be constants. After integrating the first equation of (3.23), we have

$$
a(t)=\left(K t+a_{0}\right)^{1 / 2}
$$


where $K$ and $a_{0}$ are constants. When $K>0$ and $a_{0}>0$, the plates move apart symmetrically with respect to $\eta=0$. In addition, when $K<0$ and $a_{0}>0$, the plates approach each other and squeezing flow exists with similar velocity profile as long as $\left(K t+a_{0}\right)>0$. From (3.23) and (3.24), it follows that $Q=-1$, and (3.21) becomes

$$
f^{i v}+K^{2} f^{\prime \prime}=L\left((f-\eta) f^{\prime \prime \prime}\right)+M\left((\eta-f) f^{v}\right)-N\left(2 f^{\prime \prime} f^{\prime \prime \prime}+f^{\prime} f^{i v}\right)
$$

where

$$
\begin{gathered}
K=\sqrt{\frac{3 R_{e}}{1-5 W_{e 1}}}, \\
L=\frac{R_{e}}{1-5 W_{e 1}}, \\
M=\frac{W_{e 1}}{1-5 W_{e 1}}, \\
N=\frac{W_{e 1}+W_{e 2}}{1-5 W_{e 1}} .
\end{gathered}
$$

This nonlinear boundary value problem is solved by the homotopy analysis method as well as numerically in the following sections.

\section{Basic Idea of the HAM}

Let us consider the following differential equation

$$
\mathcal{N}[f(\eta)]=0,
$$

where $\mathcal{N}$ is a nonlinear operator, $\eta$ denotes independent variable, $f(\eta)$ is an unknown function, respectively. For simplicity, we ignore all boundary or initial conditions, which can be treated in the similar way. By means of generalizing the traditional HAM, Liao [30] constructs the so-called zero-order deformation equation

$$
(1-p) \mathcal{L}\left[\varphi(\eta ; p)-f_{0}(\eta)\right]=p \hbar H(\eta) \mathcal{N}[\varphi(\eta ; p)]
$$

where $p \in[0,1]$ is the embedding parameter, $\hbar \neq 0$ is a nonzero auxiliary parameter, $H(\eta) \neq 0$ is an auxiliary function, $\mathcal{\perp}$ is an auxiliary linear operator, $f_{0}(\eta)$ is an initial guess of $f(\eta), \varphi(\eta ; p)$ is a unknown function, respectively. It is important, that one has great freedom to choose auxiliary things in the HAM. Obviously, when $p=0$ and $p=1$, it holds

$$
\varphi(\eta ; 0)=f_{0}(\eta), \quad \varphi(\eta ; 1)=u(\eta)
$$


respectively. Thus as $p$ increases from 0 to 1 , the solution $\varphi(\eta ; p)$ varies from the initial guess $f_{0}(\eta)$ to the solution $f(\eta)$. Expanding $\varphi(\eta ; p)$ in Taylor series with respect to $p$, we have

$$
\varphi(\eta ; p)=f_{0}(\eta)+\sum_{m=1}^{+\infty} f_{m}(\eta) p^{m}
$$

where

$$
f_{m}(\eta)=\left.\frac{1}{m !} \frac{\partial^{m} \varphi(\eta ; p)}{\partial p^{m}}\right|_{p=0}
$$

If the auxiliary linear operator, the initial guess, the auxiliary parameter $\hbar$, and the auxiliary function are so properly chosen, the series (4.4) converges at $p=1$, then we have

$$
f(\eta)=f_{0}(\eta)+\sum_{m=1}^{+\infty} f(\eta)
$$

which must be one of solutions of original nonlinear equation, as proved by Liao [30]. As $\hbar=-1$ and $H(\eta)=1,(4.2)$ becomes

$$
(1-p) \mathcal{L}\left[\varphi(\eta ; p)-f_{0}(\eta)\right]+p \mathcal{N}[\varphi(\eta ; p)]=0
$$

which is used mostly in the homotopy analysis method, where as the solution obtained directly, without using Taylor series [38].

According to the definition (4.5), the governing equation can be deduced from the zero-order deformation equation (4.2). Define the vector

$$
\vec{f}_{n}(\eta)=\left\{f_{0}(\eta), f_{1}(\eta), \ldots, f_{n}(\eta)\right\}
$$

Differentiating (4.2) $m$ times with respect to the embedding parameter $p$ and then setting $p=0$ and finally dividing them by $m$ !, we have the so-called $m$ th-order deformation equation

$$
\mathcal{L}\left[f_{m}(\eta)-\chi_{m} f_{m-1}(\eta)\right]=\hbar H(\eta) R_{m}\left(\vec{f}_{m-1}\right)
$$

where

$$
\begin{gathered}
R_{m}\left(\vec{f}_{m-1}\right)=\left.\frac{1}{(m-1) !} \frac{\partial^{m-1} \mathcal{N}[\varphi(\eta ; p)]}{\partial p^{m-1}}\right|_{p=0}, \\
X_{m}= \begin{cases}0, & m \leq 1, \\
1, & m>1 .\end{cases}
\end{gathered}
$$


It should be emphasized that $f_{m}(\eta)$ for $m \geq 1$ is governed by the linear equation (4.9) with the linear boundary conditions that come from original problem, which can be easily solved by symbolic computation software such as Maple and Mathematica.

\section{Application}

Consider (3.22) and (3.25) and let us solve them through the HAM. Following the HAM, we start with initial approximation $f_{0}(\eta)=-0.5 \eta^{3}+1.5 \eta$ and the linear operator

$$
\mathcal{L}[f(\eta)]=\frac{\partial^{4} \varphi(\eta ; p)}{\partial \eta^{4}}
$$

and nonlinear operator is defined as

$$
\begin{aligned}
\mathcal{N}[f(\eta)]= & -\frac{\partial^{4} f}{\partial \eta^{4}}-K^{2} \frac{\partial^{2} f}{\partial \eta^{2}}+L\left((f-\eta) \frac{\partial^{3} f}{\partial \eta^{3}}\right)+M\left((\eta-f) \frac{\partial^{5} f}{\partial \eta^{5}}\right) \\
& -N\left(2 \frac{\partial^{2} f}{\partial \eta^{2}} \frac{\partial^{3} f}{\partial \eta^{3}}+\frac{\partial f}{\partial \eta} \frac{\partial^{4} f}{\partial \eta^{4}}\right) .
\end{aligned}
$$

Using the above definition, with assumption $H(\eta)=1$, we construct the zero-order deformation equation

$$
(1-p) \mathcal{L}\left[\varphi(\eta ; p)-f_{0}(\eta)\right]=p \hbar \mathcal{N}[\varphi(\eta ; p)]
$$

Obviously, when $p=0$ and $p=1$,

$$
\varphi(\eta ; 0)=f_{0}(\eta), \quad \varphi(\eta ; 1)=f(\eta) .
$$

Differentiating the zero-order deformation equation (5.3) $m$ times with respect to $p$, and finally dividing by $m !$, we have the $m$ th-order deformation equation

$$
\mathcal{L}\left[f_{m}(\eta)-\chi_{m} f_{m-1}(\eta)\right]=\hbar R_{m}\left(\vec{f}_{m-1}\right)
$$

where

$$
\begin{aligned}
R\left(f_{m-1}\right)= & -\frac{\partial^{4} f_{m-1}(\eta)}{\partial \eta^{4}}-K^{2} \frac{\partial^{2} f_{m-1}(\eta)}{\partial \eta^{2}}-L \eta \frac{\partial^{3} f_{m-1}(\eta)}{\partial \eta^{3}}+M \eta \frac{\partial^{5} f_{m-1}(\eta)}{\partial \eta^{5}} \\
& +L \sum_{n=0}^{m-1} f_{n}(\eta) \frac{\partial^{3} f_{m-1-n}(\eta)}{\partial \eta^{3}}-M \sum_{n=0}^{m-1} f_{n}(\eta) \frac{\partial^{5} f_{m-1-n}(\eta)}{\partial \eta^{5}} \\
& -2 N \sum_{n=0}^{m-1} \frac{\partial^{2} f_{n}(\eta)}{\partial \eta^{2}} \frac{\partial^{3} f_{m-1-n}(\eta)}{\partial \eta^{3}}-N \sum_{n=0}^{m-1} \frac{\partial f_{n}(\eta)}{\partial \eta} \frac{\partial^{4} f_{m-1-n}(\eta)}{\partial \eta^{4}} .
\end{aligned}
$$


Then

$$
\begin{gathered}
\mathcal{L}\left[f_{m}(\eta)-x_{m} f_{m-1}(\eta)\right]=\hbar R_{m}\left(\vec{f}_{m-1}\right), \\
\chi_{m}= \begin{cases}0, & m \leq 1, \\
1, & m>1 .\end{cases}
\end{gathered}
$$

We choose auxiliary functions as follow:

$$
\mathscr{\ell}(\eta)=1
$$

Following the HAM and trying higher iterations with the unique and proper assignment of the results converge to the exact solution:

$$
f(\eta) \approx f_{0}(\eta)+f_{1}(\eta)+f_{2}(\eta)+\cdots+f_{m}(\eta)
$$

using the symbolic software Mathematica to solve the system of linear equations, (5.5), with the boundary conditions (3.22), and successively obtain

$$
f_{1}(\eta)=-0.058567 \hbar \eta+0.115348 \hbar \eta^{3}-0.054996 \hbar \eta^{5}-0.001786 \hbar \eta^{7}
$$

\section{Results and Discussion}

In this work the unsteady 2-dimensional flow of nonconducting, incompressible second grade fluid between two circular plates is considered. The squeezing flow is generated by moving the plates symmetrically with respect to the central region $z=0$. The unsteady equations of motion are reduced to a single nonlinear 5 th-order ordinary differential by using a similarity transformation. The resulting nonlinear boundary value problem is solved using the homotopy analysis method and numerical technique. The effect of the Reynolds number $R_{e}$ on velocity profiles is depicted. In these profiles we fixed the nonNewtonian parameters $W_{e 1}=0.0, W_{e 2}=0.05$ and varied $R_{e}$ as $R_{e}=1.0,1.5,2.0$ (Figures 11 and 12). It is noted that the normal velocity increases as the Reynolds number increases. It is also observed that at a given time and for a fixed positive value of Reynolds number the normal velocity increases monotonically from $\eta=0$ to $\eta=1$ presents effect of Reynolds number on the longitudinal velocity. It is observed that this component of velocity increases near the central axis of the channel but deceases near the walls. The influence of nonNewtonian parameter $W_{e 1}$ on the normal and longitudinal velocity components is depicted. we varied $W_{e 1}=0.0,0.05,0.75$ fixing $W_{e 2}=0.05, R_{e}=1.0$. This observation on the velocity components is similar to that of Reynolds number $R_{e}$ presents the effects of nonNewtonian parameter $W_{e 2}$ on the squeezing flow of the second grade fluid. It is noted that effect of $W_{e 2}$ on the velocity components is opposite to that of the nonNewtonian parameter $W_{e 1}$.

The HAM was applied successfully to find an explicit, totally analytic, uniformly valid solution for unsteady squeezing flow between circular parallel plates. The validity of our analytic solutions is verified by numerical results. The results show that HAM is very accurate (Figures 3, 4, 6, 7, 9, and 10). Also, the HAM gives rapidly convergent series with specific significant features for each scheme (Figures 2, 5, and 8). The accuracy of the method is very good. 


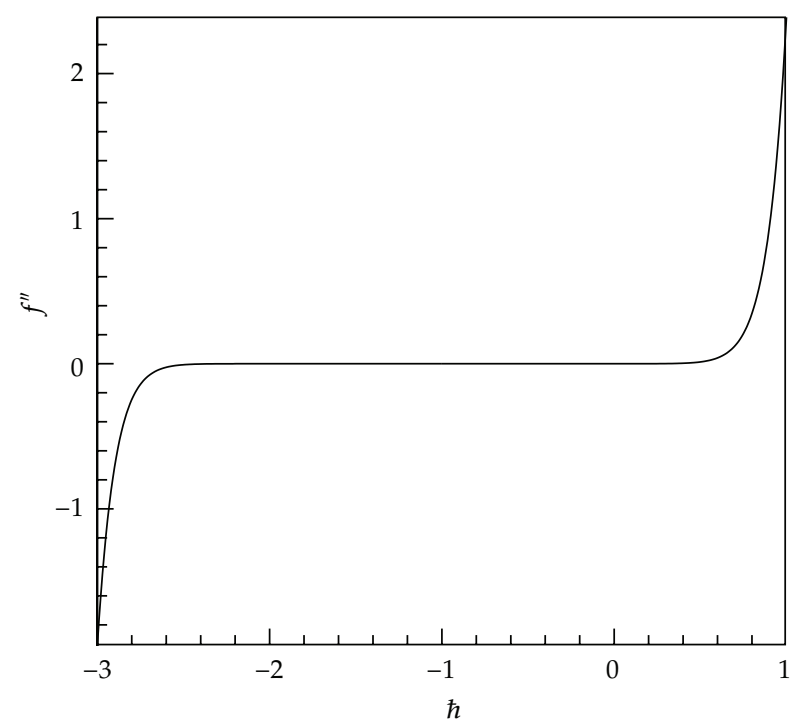

Figure 2: $\hbar$ curve by the HAM with $x=0.00001, K=1.732, L=1, M=0$, and $N=0.05$, by 20th-order approximation solution.

\section{Conclusions}

A similarity solution of the problem for the unsteady flow of nonconducting, incompressible second grade fluid between two circular plates approaching or receding from each other has been investigated. It is noted that a similarity solution exists only when the distance between the plates varies as $\left(K t+a_{0}\right)^{1 / 2}$, and squeezing flow takes place for $K<0$ and $a_{0}>0$ as for as $K t+a_{0}>0$, Approximate solutions for the fluid velocity have been found for the flow of unsteady 2-dimensional second grade fluid between two circular plates by the homotopy analysis method and numerical method. The major finding of the present paper can be summarized as follows:

The Reynolds number $R_{e}$ and nonNewtonian parameter $W_{e 1}$ have similar effect on the normal and longitudinal velocity components.

The nonNewtonian parameter $W_{e 2}$ has opposite trend on the velocity profiles to that of Reynolds number and $W_{e 1}$.

It is found that at a given time and for a fixed positive value of Reynolds number, nonNewtonian parameters $W_{e 1}, W_{e 2}$, the normal velocity increases monotonically from $\eta=0$ to $\eta=1$.

The longitudinal component of velocity increases near the central axis of the channel but deceases near the walls when $R_{e}$ increases from 1.0 to 2.0. Similar effect of $W_{e 2}$ on this component is observed when $W_{e 2}$ varies from 0.0 to 0.1 .

The results are recovered for $W_{e} \rightarrow 0$.

The obtained results are valid for all values of Reynolds number and nonNewtonian parameters. 


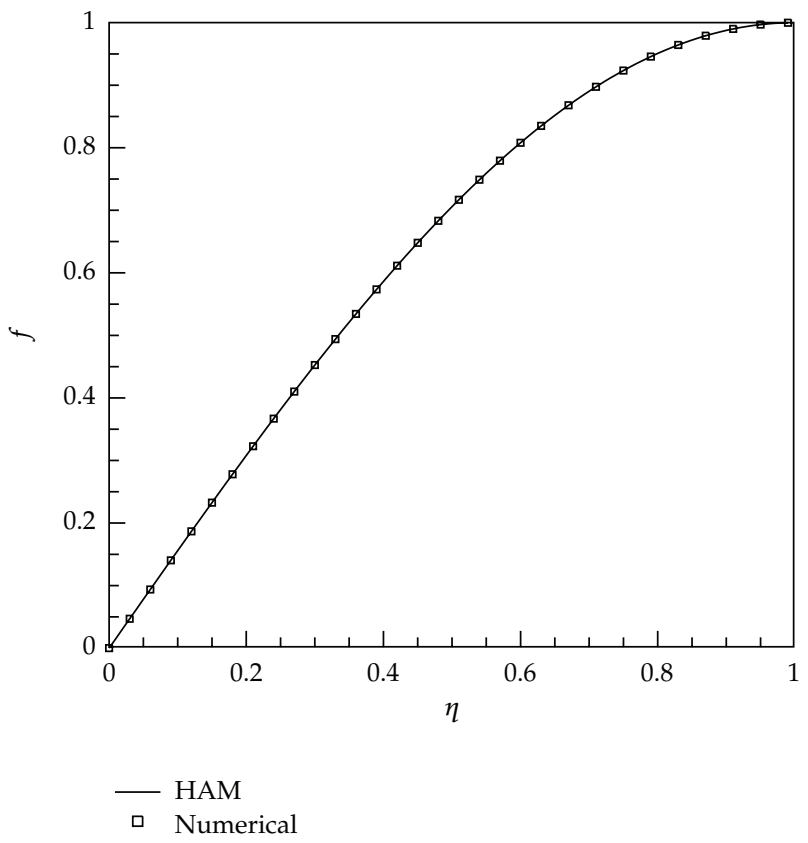

Figure 3: Obtained solution by the HAM with $\hbar=-1, K=1.732, L=1, M=0$, and $N=0.05$, by 20th-order approximation solution.

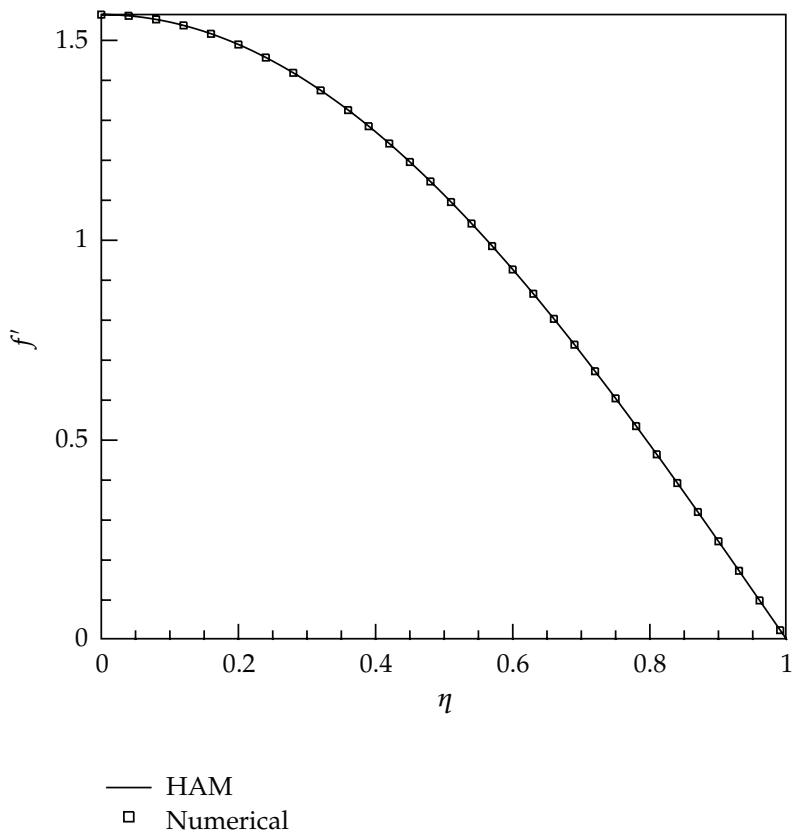

Figure 4: Obtained solution by the HAM with $\hbar=-1, K=1.732, L=1, M=0$, and $N=0.05$, by 20th-order approximation solution. 


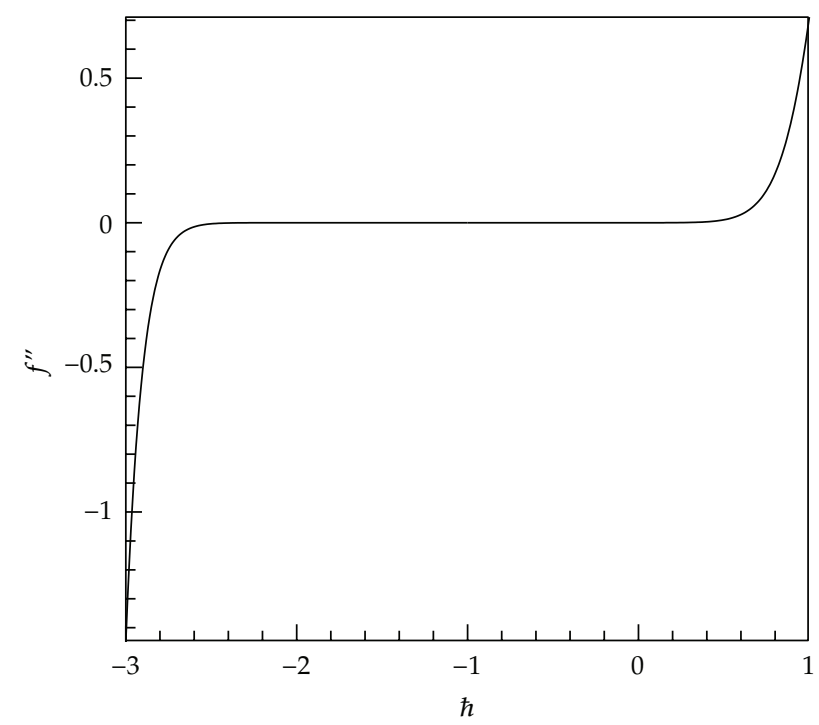

Figure 5: $\hbar$ curve by the HAM with $x=0.00001, K=2.121, L=1.5, M=0$, and $N=0.05$, by 20th-order approximation solution.

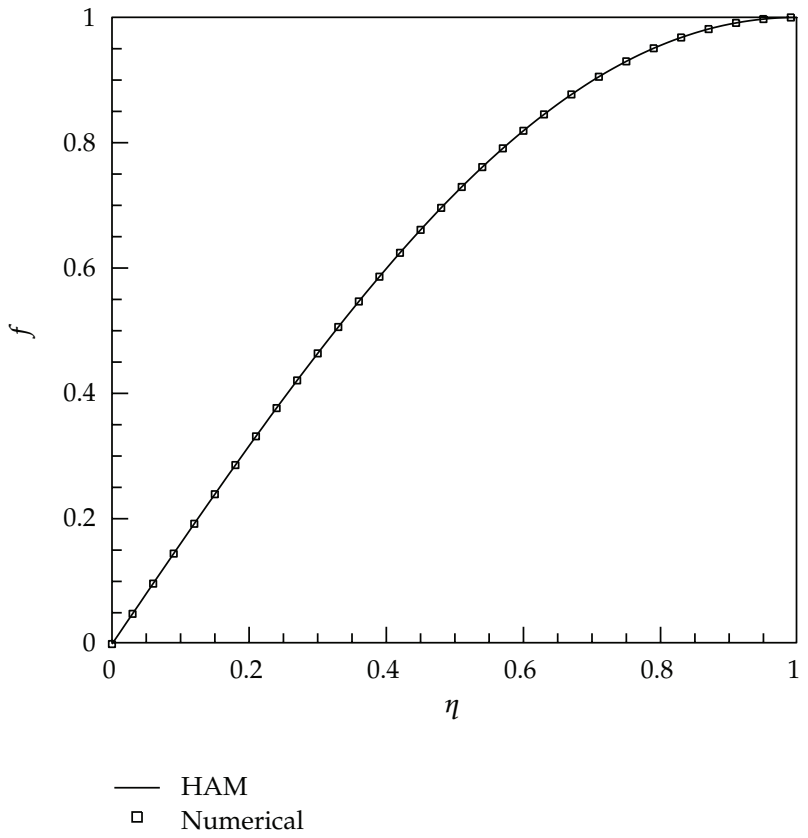

Figure 6: Obtained solution by the HAM with $\hbar=-1, K=2.121, L=1.5, M=0$, and $N=0.05$, by 20th-order approximation solution. 


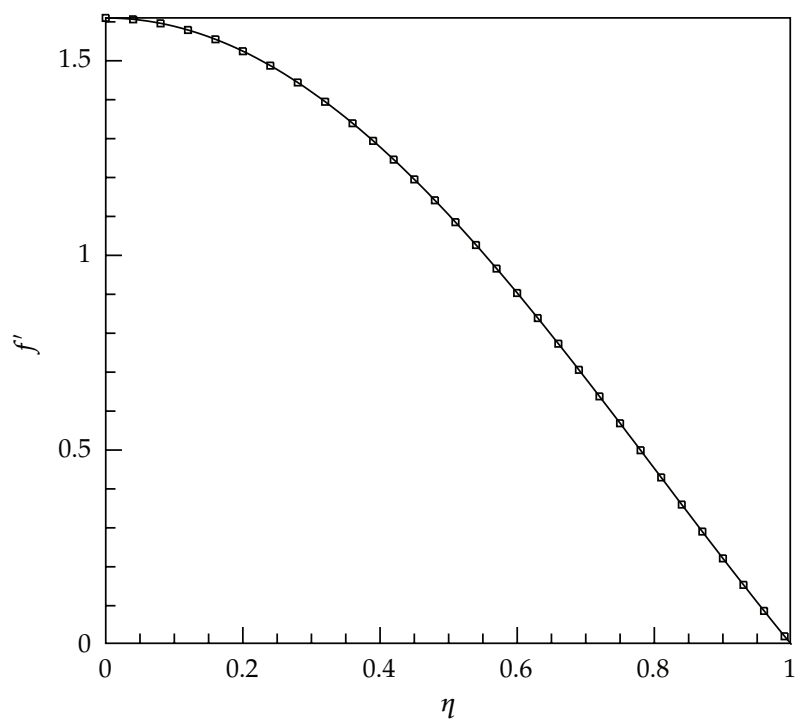

$\begin{array}{ll}\text { - } & \text { HAM } \\ & \text { Numerical }\end{array}$

Figure 7: Obtained solution by the HAM with $\hbar=-1, K=2.121, L=1.5, M=0$, and $N=0.05$, by 20th-order approximation solution.

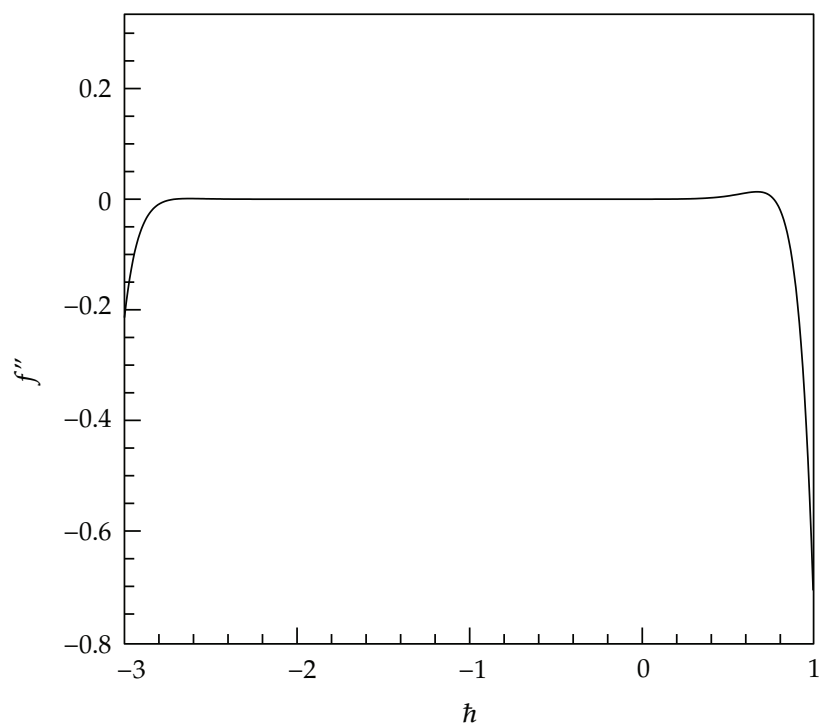

Figure 8: $\hbar$ curve by the HAM with $x=0.00001, K=2.449, L=2, M=0$, and $N=0.05$, by 10th-order approximation solution. 


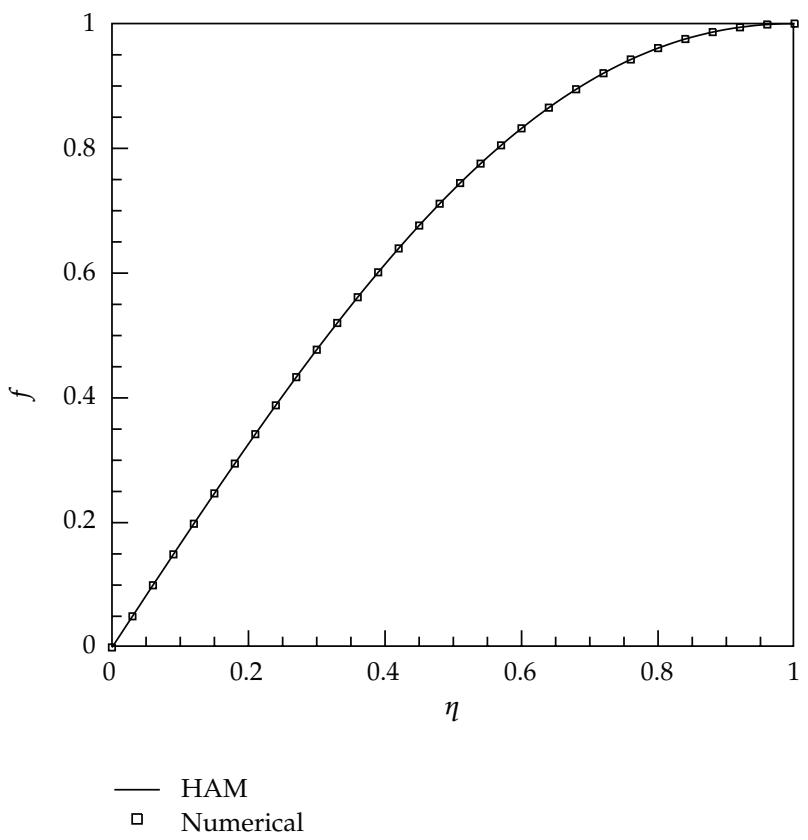

Figure 9: Obtained solution by the HAM with $\hbar=-1, K=2.449, L=2, M=0$, and $N=0.05$, by 20th-order approximation solution.

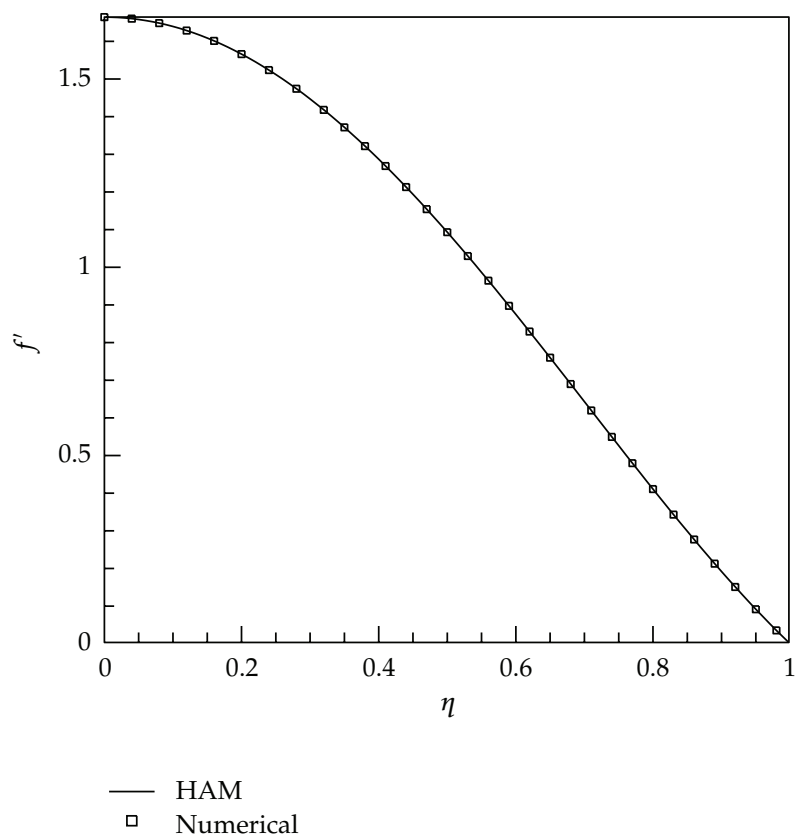

Figure 10: Obtained solution by the HAM with $\hbar=-1, K=2.449, L=2, M=0$, and $N=0.05$, by 20th-order approximation solution. 


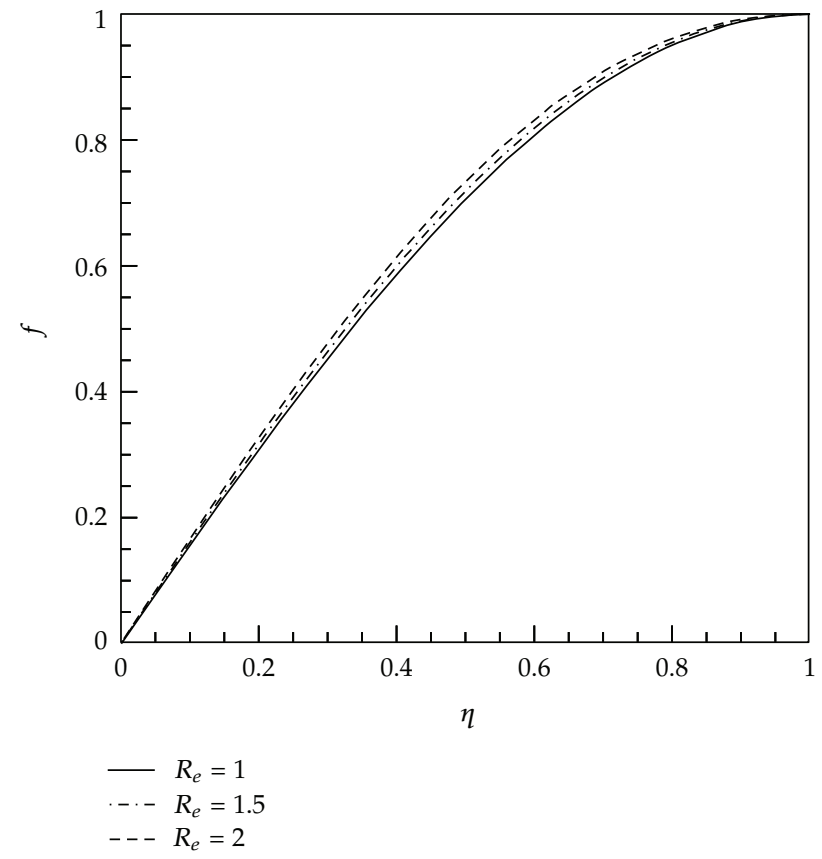

Figure 11: The effect of $R_{e}$ on the axial velocity profiles.

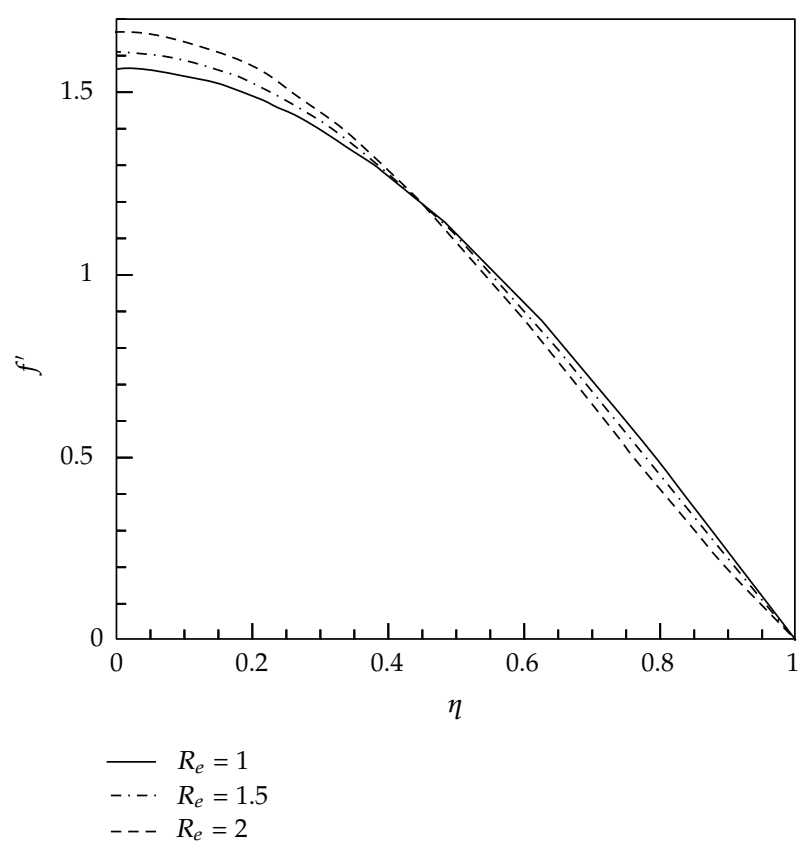

Figure 12: The effect of $R_{e}$ on the radial velocity profiles. 


\title{
Nomenclature
}

\author{
$\rho: \quad$ Density \\ $v: \quad$ Kinematic viscosity \\ $V: \quad$ Velocity vector \\ $P: \quad$ Pressure \\ $T: \quad$ Stress tensor \\ $\mu$ : $\quad$ Viscosity coefficient \\ $\alpha_{1}: \quad$ Material constant \\ $\alpha_{2}$ : Material constant \\ $\Omega: \quad$ Vorticity function \\ $h$ : $\quad$ Pressure gradient \\ $\nabla^{2}$ : $\quad$ Laplacian operator \\ $\eta$ : $\quad$ Dimensionless variable \\ $\hbar$ : Auxiliary linear parameter \\ $A_{i}$ : Rivlin-Ericksen tensor \\ $u$ : Velocity component along the radial \\ $w$ : Velocity component along the axial \\ $2 a(t)$ : Distance between the plates at any time $t$ \\ $W_{e 1}$ : Non-Newton parameter \\ $W_{e 2}$ : Non-Newton parameter \\ $R_{e}$ : Reynolds number.
}

\section{References}

[1] M. J. Stefan, "Versuch Uber die scheinbare adhesion," Akademie der Wissenschaften in Wien. MathematikNaturwissen, vol. 69, p. 713, 1874.

[2] J. J. Bickerman, Development in Theoretical and Applied Mathematics, vol. 3, Academic Press, New York, NY, USA, 1958

[3] D. Yao, V. L. Virupaksha, and B. Kim, "Study on squeezing flow during nonisothermal embossing of polymer microstructures," Polymer Engineering and Science, vol. 45, no. 5, pp. 652-660, 2005.

[4] W. E. Langlois, "Isothermal squeeze films," Applied Mathematics, vol. 20, p. 131, 1962.

[5] E. O. Salbu, "Compressible squeeze films and squeeze bearings," Journal of Basic Engineering, vol. 86, p. 355, 1964.

[6] J. F. Thorpe, in Development in Theoretical and Applied Mathematics, W. A. Shah, Ed., vol. 3, Pergamon Press, Oxford, UK, 1967.

[7] P. S. Gupta and A. S. Gupta, "Squeezing flow between parallel plates," Wear, vol. 45, no. 2, pp. 177$185,1977$.

[8] A. F. Elkouh, "Fluid inertia effects in a squeeze film between two plane annuli," Journal of Tribology, vol. 106, no. 2, pp. 223-227, 1984.

[9] R. L. Verma, “A numerical solution for squeezing flow between parallel channels," Wear, vol. 72, no. 1, pp. 89-95, 1981.

[10] P. Singh, V. Radhakrishnan, and K. A. Narayan, "Squeezing flow between parallel plates," IngenieurArchiv, vol. 60, no. 4, pp. 274-281, 1990.

[11] E. A. Hamza, "Suction and injection effects on a similar flow between parallel plates," Journal of Physics D: Applied Physics, vol. 32, no. 6, pp. 656-663, 1999.

[12] K. R. Rajagopal and A. S. Gupta, "On a class of exact solutions to the equations of motion of a second grade fluid," International Journal of Engineering Science, vol. 19, no. 7, pp. 1009-1014, 1981.

[13] B. S. Dandapat and A. S. Gupta, "Stability of a thin layer of a second-grade fluid on a rotating disk," International Journal of Non-Linear Mechanics, vol. 26, no. 3-4, pp. 409-417, 1991.

[14] R. M. Barron and J. T. Wiley, "Newtonian flow theory for slender bodies in a dusty gas," Journal of Fluid Mechanics, vol. 108, pp. 147-157, 1981.

[15] A. K. Ghosh and L. Debnath, "Hydromagnetic Stokes flow in a rotating fluid with suspended small particles," Applied Scientific Research, vol. 43, no. 3, pp. 165-192, 1986. 
[16] Lokenath Debnath and A. K. Ghosh, "On unsteady hydromagnetic flows of a dusty fluid between two oscillating plates," Applied Scientific Research, vol. 45, no. 4, pp. 353-365, 1988.

[17] M. H. Hamdan and R. M. Barron, "Analysis of the squeezing flow of dusty fluids," Applied Scientific Research, vol. 49, no. 4, pp. 345-354, 1992.

[18] R. J. Grimm, "Squeezing flows of Newtonian liquid films an analysis including fluid inertia," Applied Scientific Research, vol. 32, no. 2, pp. 149-166, 1976.

[19] W. A. Wolfe, "Squeeze film pressures," Applied Scientific Research, vol. 14, no. 1, pp. 77-90, 1965.

[20] D. C. Kuzma, "Fluid inertia effects in squeeze films," Applied Scientific Research, vol. 18, no. 1, pp. 15-20, 1968.

[21] R. J. Grimm, "Squeezing flows of Newtonian liquid films an analysis including fluid inertia," Applied Scientific Research, vol. 32, no. 2, pp. 149-166, 1976.

[22] . TICHY JA and . WINER WO, "Inertial considerations in parallel circular squeeze film bearings," Transactions of the ASME: Journal of Lubrication Technology, vol. 92, pp. 588-592, 1970.

[23] C. Y. Wang and L. T. Watson, "Squeezing of a viscous fluid between elliptic plates," Applied Scientific Research, vol. 35, no. 2-3, pp. 195-207, 1979.

[24] R. Usha and R. Sridharan, "Arbitrary squeezing of a viscous fluid between elliptic plates," Fluid Dynamics Research, vol. 18, no. 1, pp. 35-51, 1996.

[25] H. M. Laun, M. Rady, and O. Hassager, "Analytical solutions for squeeze flow with partial wall slip," Journal of Non-Newtonian Fluid Mechanics, vol. 81, no. 1-2, pp. 1-15, 1999.

[26] M. H. Hamdan and R. M. Barron, "Analysis of the squeezing flow of dusty fluids," Applied Scientific Research, vol. 49, no. 4, pp. 345-354, 1992.

[27] P. T. Nhan, "Squeeze flow of a viscoelastic solid," Journal of Non-Newtonian Fluid Mechanics, vol. 95, pp. 43-362, 2000.

[28] S. J. Liao, The proposed homotopy analysis technique for the solution of nonlinear problems, Ph.D. thesis, Shanghai Jiao Tong University, 1992.

[29] S.-J. Liao, "An explicit, totally analytic approximate solution for Blasius' viscous flow problems," International Journal of Non-Linear Mechanics, vol. 34, no. 4, pp. 759-778, 1999.

[30] S.-J. Liao, "On the analytic solution of magnetohydrodynamic flows of non-Newtonian fluids over a stretching sheet," Journal of Fluid Mechanics, vol. 488, pp. 189-212, 2003.

[31] S. J. Liao, Beyond Perturbation, vol. 2 of Introduction to the Homotopy Analysis Method, Chapman \& Hall/CRC, Boca Raton, FL, 2004.

[32] S. J. Liao, "On the homotopy analysis method for nonlinear problems," Applied Mathematics and Computation, vol. 147, no. 2, pp. 499-513, 2004.

[33] S. Liao, "A new branch of solutions of boundary-layer flows over an impermeable stretched plate," International Journal of Heat and Mass Transfer, vol. 48, no. 12, pp. 2529-2539, 2005.

[34] S. Liao, "Comparison between the homotopy analysis method and homotopy perturbation method," Applied Mathematics and Computation, vol. 169, no. 2, pp. 1186-1194, 2005.

[35] S. Liao, J. Su, and A. T. Chwang, "Series solutions for a nonlinear model of combined convective and radiative cooling of a spherical body," International Journal of Heat and Mass Transfer, vol. 49, no. 15-16, pp. 2437-2445, 2006.

[36] M. M. Rashidi and S. A. Mohimanian Pour, "Analytic approximate solutions for unsteady boundarylayer flow and heat transfer due to a stretching sheet by homotopy analysis method," Nonlinear Analysis. Modelling and Control, vol. 15, no. 1, pp. 83-95, 2010.

[37] M. M. Rashidi, D. D. Ganji, and S. Dinarvand, "Approximate traveling wave solutions of coupled Whitham-Broer-Kaup shallow water equations by homotopy analysis method," Differential Equations $\mathcal{E}$ Nonlinear Mechanics, vol. 2008, Article ID 243459, 8 pages, 2008.

[38] M. M. Rashidi, G. Domairry, and S. Dinarvand, "Approximate solutions for the Burger and regularized long wave equations by means of the homotopy analysis method," Communications in Nonlinear Science and Numerical Simulation, vol. 14, no. 3, pp. 708-717, 2009.

[39] G. Birkhoff, Hydrodynamics, Princeton University Press, Princeton, NJ, USA, 1960. 


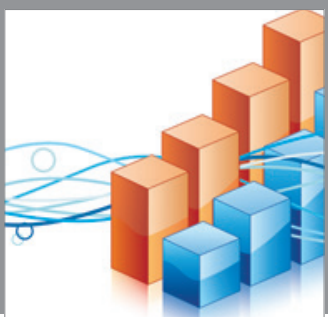

Advances in

Operations Research

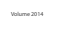

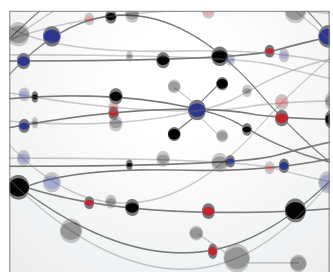

\section{The Scientific} World Journal
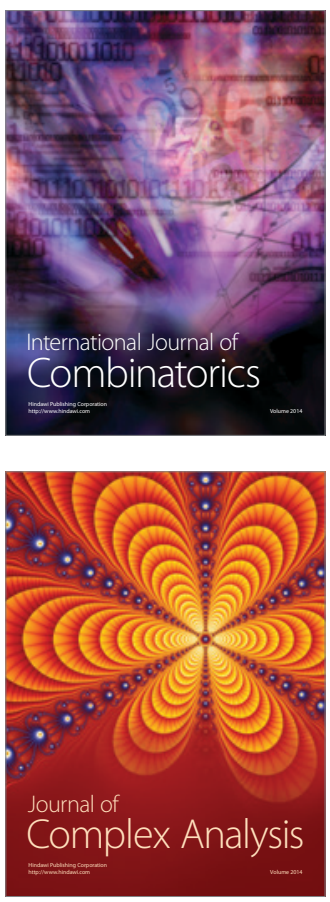

International Journal of

Mathematics and

Mathematical

Sciences
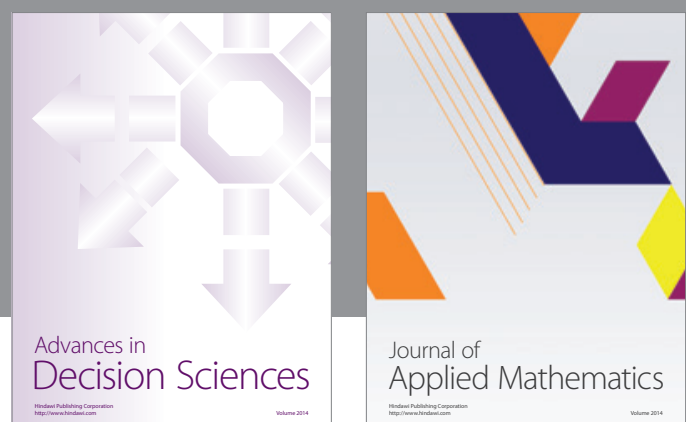

Journal of

Applied Mathematics
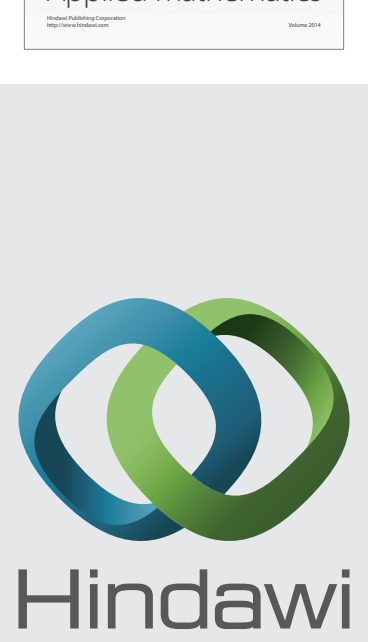

Submit your manuscripts at http://www.hindawi.com
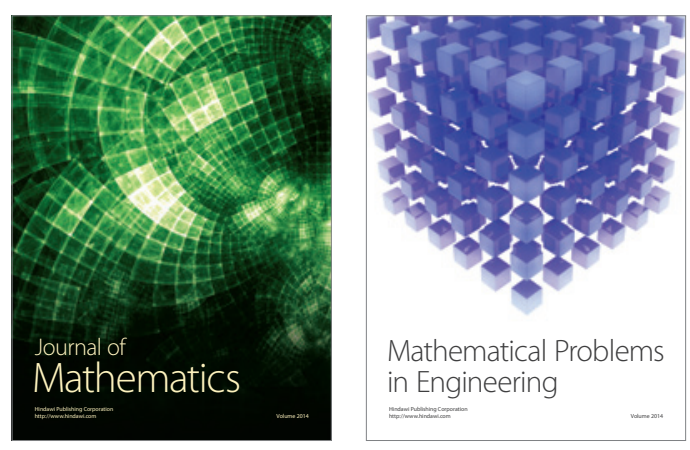

Mathematical Problems in Engineering
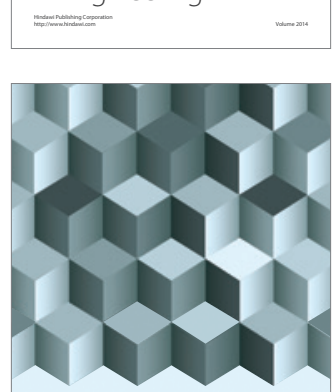

Journal of

Function Spaces
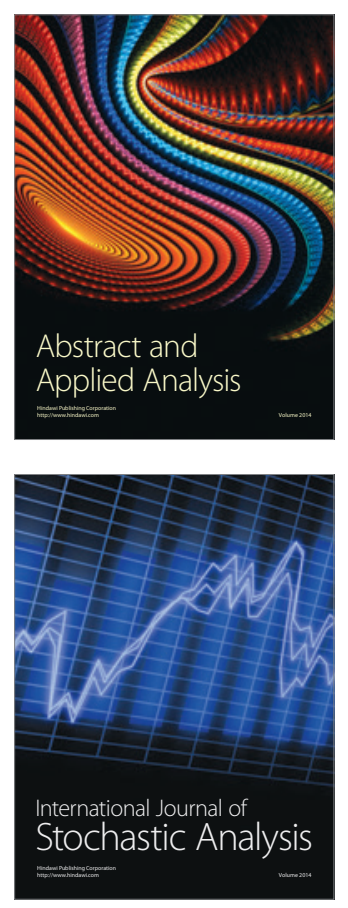

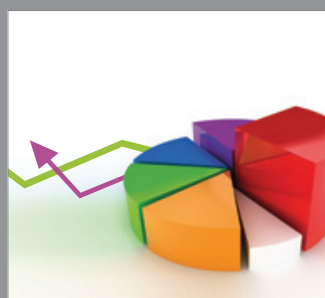

ournal of

Probability and Statistics

Promensencen
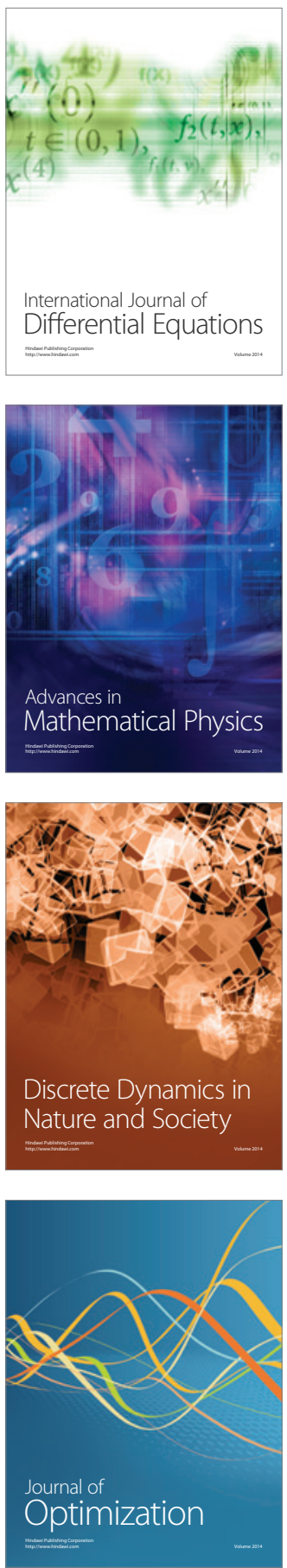